\title{
Harmonized Landsat/Sentinel-2 Products for Land Monitoring
}

\author{
Jeffrey Masek \\ Biospheric Sciences Laboratory \\ NASA GSFC \\ Greenbelt, MD USA \\ Jeffrey.G.Masek@nasa.gov
}

\author{
Junchang Ju \\ Earth System Science \\ Interdisciplinary University of \\ Maryland \\ College Park, MD USA \\ Junchang.Ju@nasa.gov \\ Martin Claverie \\ Earth and Life Institute \\ Universite Catholique de Louvain \\ Louvain, Belgium \\ Martin.Claverie@gmail.com
}

\author{
Jean-Claude Roger \\ Dept. Geographical Sciences \\ University of Maryland \\ College Park, MD USA \\ Jean-Claude.Roger@nasa.gov
}

\author{
Sergii Skakun \\ Dept. Geographical Sciences \\ University of Maryland, line 4: \\ College Park, MD USA \\ sergii.skakun@nasa.gov
}

\author{
Jennifer Dungan \\ Earth Science Division \\ NASA Ames Research Center \\ Moffitt Field, CA USA \\ Jennifer.L.Dungan@nasa.gov
}

\begin{abstract}
The Harmonized Landsat-8 and Sentinel-2 (HLS) project is a NASA initiative aiming to produce a seamless, harmonized surface reflectance record from the Operational Land Imager (OLI) and Multi-Spectral Instrument (MSI) aboard Landsat-8 and Sentinel-2 remote sensing satellites, respectively. The HLS products are based on a set of algorithms to obtain seamless products from both sensors (OLI and MSI): atmospheric correction, cloud and cloud-shadow masking, geographic co-registration and common gridding, bidirectional reflectance distribution function normalization and bandpass adjustment. As of version 1.3, the HLS v1.3 data set covers 9.12 million $\mathrm{km} 2$ and spans from first Landsat-8 data (2013) to present. HLS products provide near-daily surface reflectance information with a common geometric framework, and are suitable for a variety of agricultural and vegetation monitoring tasks, including analysis of crop type, condition, and phenology.
\end{abstract}

Keywords-land remote sensing, Landsat, Sentinel-2, atmospheric correction

\section{INTRODUCTION}

Many land monitoring applications require more frequent observations than can be obtained from a single "Landsatclass" sensor. Examples of these applications include crop type and condition monitoring, vegetation phenology, disaster response, and surface water quality. These and numerous other applications all require near-daily imagery at medium spatial resolution. Even year-to-year change detection benefits from frequent data coverage in cloudy areas [1]. Furthermore, with the advent of free imagery archives $[2,3,4]$ and the increased availability of powerful computing environments, researchers have developed a new capacity for working with large volumes of time-series imagery.

Since trying to achieve near-daily observation frequency from a single, well-calibrated satellite system may be prohibitively expensive, a reasonable alternative is to combine data from multiple international sources. The Harmonized Landsat/Sentinel-2 (HLS) Project is working toward this goal by generating a seamless surface reflectance product from Landsat 8 and Sentinel-2 inputs. Specifically, by "harmonized" we mean that the products are:

- Gridded to a common pixel resolution, projection, and spatial extent (i.e., tile);

- Atmospherically corrected to surface reflectance using a common radiative transfer algorithm [5];

- Normalized to a common nadir view geometry via Bidirectional Reflectance Distribution Function (BRDF) estimation [6];

- $\quad$ Adjusted to represent the response from a common spectral bandpass.

In essence these products are the building blocks for a "data cube" such that a user may examine any given pixel through time, and treat the near-daily reflectance time series as though it came from a single sensor

\section{PRODUCT DESCRIPTION AND ALGORITHMS}

\section{A. Product Flow}

The overall product flow is shown in Fig. 1. Input data products from Landsat 8 (Collection 1 or L1T top-ofatmosphere) and Sentinel-2 (L1C top-of-atmosphere) are ingested for HLS processing. A series of radiometric and geometric corrections are applied (see below). Three types of products are then generated: "S10" products - atmospherically corrected Sentinel-2 images in their native resolution and geometry; and the harmonized products "S30" and "L30". These products have been radiometrically harmonized to the 
maximum extent, and then gridded to a common 30-meter UTM basis using the Sentinel-2 tile system.

\section{B. Algorithm Descriptions}

Atmospheric Correction: The atmospheric correction method is based on the Land Surface Reflectance Code (LaSRC), an algorithm primarily developed for operational use with Landsat-8 imagery [5]. In brief, LaSRC assumes a Lambertian, plane-parallel atmosphere, and uses the $6 \mathrm{~S}$ radiative transfer model to invert directional surface spectral reflectance from observed top-of-atmosphere reflectance. The retrieval accounts for both molecular and particle scattering, as well as absorption by water vapor and ozone. Uncertainty estimates for LaSRC are based on comparison with corrections based on in-situ atmospheric parameters from the Aerosol Robotic Network (AERONET, [5]). For Landsat-8 OLI, overall uncertainty varied from $0.11 \%$ absolute reflectance (SWIR1 band) to $0.85 \%$ absolute reflectance (blue band). A separate version of LaSRC has been prepared for the use with Sentinel-2/MSI imagery, incorporating pre-launch measurements of the MSI spectral band-passes.

Cloud Masking: Cloud and shadow masks are essential for use of products in automated analysis. HLS uses a set of cloud masks to minimize errors of cloud omission. The LaSRC package includes a cloud and shadow mask for both Landsat 8 and Sentinel-2. For S30 products, HLS uses the union of those masks with the Boston University Fmask algorithm [7,8] adapted for Sentinel-2. For L30 products, HLS uses the union of the USGS cloud QA bits and the LaSRC mask.

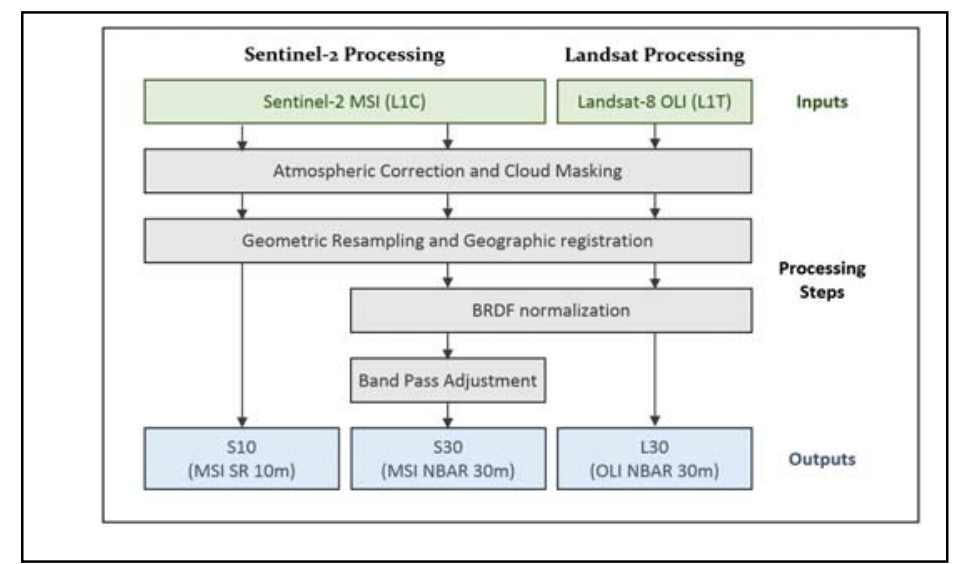

Fig. 1: HLS Product and Algorithm Flow

BRDF Correction: Given the differing solar and view angles associated with Landsat- 8 and Sentinel-2, normalizing the BRDF effects is desirable. Roy et al. [6] have highlighted that a single, global and constant BRDF shape produces satisfying BRDF normalization over a limited range of view zenith angle near nadir. We selected the c-factor technique and global coefficients provided by Roy et al. [6] because the technique is very stable, reversible, easy to implement for operational processing and have been evaluated for Sentinel-2 data [6,9]. The S30 and L30 reflectance products are normalized for per-pixel view and per-tile illumination angles.

\footnotetext{
Identify applicable funding agency here. If none, delete this text box.
}

This normalization is applied to all S30 and L30 optical bands except the MSI red-edge bands and the cirrus and water vapor bands for which no MODIS BRDF information are available. The view angle is set to nadir and the solar zenith angle is fixed through time but varies for each tile based on the latitude.

Spectral Bandpass Adjustment: The harmonization also requires adjustment of the small differences between the equivalent spectral bands of MSI and OLI. The OLI spectral band passes are used as reference, to which the MSI spectral bands are adjusted. No band pass adjustment is defined for (i) MSI red-edge bands (B05, B06 and B07), (ii) broad NIR band (B08), and (iii) atmospheric bands (B09 and B10). A simple least squares linear regression between equivalent spectral bands was used, as in [10].

\section{PRODUCT QUALITY ASSESSMENT}

We distinguish between product Quality Assessment (QA) and Validation. The former is geared toward generating qualitative information on the suitability of individual pixels or granules for further analysis, while the latter provides quantitative assessment of product uncertainty. HLS Product QA relies primarily on comparison with contemporary MODIS Terra Climate Modeling Grid (MOD09CMG) reflectance products. HLS granules with more than one CMG pixel that exhibit large deviations from the contemporary MODIS CMG data are flagged and discarded from the published data set. Typically we have found that $\sim 4 \%$ of S30 and $0.5 \%$ of L30 products are flagged, owing to the relatively poorer performance of the S30 cloud mask in the absence of a thermal infrared band for Sentinel-2.

Quantitative validation of the HLS reflectanc products is still in progress. We rely primarily on (i) validation of the LaSRC atmospheric correction approach as described in [5]; (ii) comparison with SURFAD broadband radiometric measurements; (iii) analysis of temporal stability over psudoinvariant calibration sites. Analysis of the stability over one desert site in Arizona suggests short-term stability better than $\sim 3.6 \%$ relative.

\section{STATUS AND FUTURE DIRECTION}

Currently version 1.3 of the HLS data set has been released, encompassing about $7 \%$ of the global land area $(9.12$ million $\mathrm{km} 2$ ). The temporal range of products is 2013-2017 for the L30 products and October 2015-2017 for the S30 products. Data have been produced for a range of test sites, ranging from individual tiles (e.g. AERONET and SURFRAD sites), to entire countries (Germany, Tanzania, South Africa). These larger regions have been generated to test specific applications. For example, Tanzania is a GEOGLAM focus site, while University of Humbolt used HLS data across Germany to produce the first crop type map for that nation. HLS processing has utilized the NASA Earth Exchanage (NEX) computing environment at NASA Ames Research Center. Products may be downloaded via anonymous FTP from the GSFC HLS web site: https://hls.gsfc.nasa.gov. 
During 2018 we intend to expand HLS production to include all of North America, and begin routine daily product generation with latency $<5$ days. Analysis of existing MODIS imagery suggests that over most mid-latitude agricultural areas, cloud-free HLS observations should be available at least weekly given the Landsat 8 and Sentinel-2a,b virtual constellation [11]. Harmonized products with this frequency would be able to support improved characterization of agricultural phenology, which in turn can be used to identify crop types, management practice, and crop condition (Fig. 2).

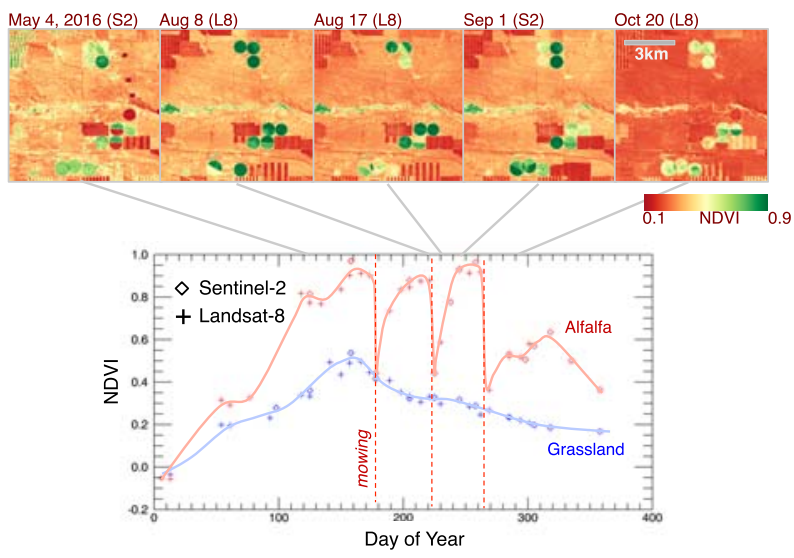

Fig. 2. Seasonal phenology (greening) for natural grassland (blue line) and irrigated alfalfa fields (red line) near Cheyenne Wyoming observed from Harmonized Landsat/Sentinel-2 data products. The high temporal density of observations allows individual mowing events to be detected within alfalfa fields.

\section{ACKNOWLEDGMENT}

This work was supported by the NASA Land Cover Project Science Office and the NASA Earth Science Research and Analysis (R\&A) Program. HLS contains modified Copernicus Sentinel data (2015-2017) processed by ESA.

\section{REFERENCES}

[1] Roy, D.P., Lewis, P., Schaaf, C.B., Devadiga, S., \& Boschetti, L. (2006). The global impact of clouds on the production of MODIS bidirectional reflectance model-based composites for terrestrial monitoring. Ieee Geoscience and Remote Sensing Letters, 3, 452-456

[2] Loveland, T.R., \& Dwyer, J.L. (2012). Landsat: Building a strong future. Remote Sensing of Environment, 122, 22-29

[3] Wulder, M.A., Masek, J.G., Cohen, W.B., Loveland, T.R., \& Woodcock, C.E. (2012). Opening the archive: How free data has enabled the science and monitoring promise of Landsat. Remote Sensing of Environment, 122, 2-1.

[4] Woodcock, C.E., Allen, R., Anderson, M., Belward, A., Bindschadler, R., Cohen, W., Gao, F., Goward, S.N., Helder, D., Helmer, E., Nemani, R., Oreopoulos, L., Schott, J., Thenkabail, P.S., Vermote, E.F., Vogelmann, J., Wulder, M.A., \& Wynne, R. (2008). Free Access to Landsat Imagery. Science, 320, 1011.

[5] Vermote, E., Justice, C., Claverie, M., \& Franch, B. (2016). Preliminary analysis of the performance of the Landsat 8/OLI land surface reflectance product. Remote Sensing of Environment

[6] Roy, D.P., Zhang, H.K., Ju, J., Gomez-Dans, J.L., Lewis, P.E., Schaaf, C.B., Sun, Q., Li, J., Huang, H., \& Kovalskyy, V. (2016). A general method to normalize Landsat reflectance data to nadir BRDF adjusted reflectance. Remote Sensing of Environment, 176, 255-271

[7] Zhu, Z., \& Woodcock, C.E. (2012). Object-based cloud and cloud shadow detection in Landsat imagery. Remote Sensing of Environment, $118,83-94$

[8] Zhu, Z., \& Woodcock, C.E. (2014). Automated cloud, cloud shadow, and snow detection in multitemporal Landsat data: An algorithm designed specifically for monitoring land cover change. Remote Sensing of Environment, 152, 217-234

[9] Claverie, M., Vermote, E., Franch, B., He, T., Hagolle, O., Kadiri, M., \& Masek, J. (2015). Evaluation of Medium Spatial Resolution BRDFAdjustment Techniques Using Multi-Angular SPOT4 (Take5) Acquisitions. Remote Sensing, 7, 12057

[10] Teillet, P.M., Fedosejevs, G., Thome, K.J., \& Barker, J.L. (2007). Impacts of spectral band difference effects on radiometric crosscalibration between satellite sensors in the solar-reflective spectral domain. Remote Sensing of Environment, 110, 393-409

[11] Whitcraft, A., Becker-Reshef, I., and Justice, C.O., A framework for defining spatial explicit earth observation requirements for a global agricultural monitoring initiative (GEOGLAM), 2015, Remote Sens. $2015,7(2), 1461-1481$ 